\title{
New Advances in Forensic Analysis of Soil Phases
}

\author{
Marek Kotrly ${ }^{1,2}$ and Ivana Turkova ${ }^{2}$
}

${ }^{1}$ Institute of Criminalistics, Prague, Czech Republic, ${ }^{2}$ Charles University in Prague, Faculty of Science, Praha, Czech Republic

Soils analysis started to be more massively applied in the field of forensic sciences in the 1970s and, at first, it was carried out in a quite easy way based only on a simple chemical analysis without any detailed comparison at the level of the individual mineral phases. Gradually, the complex system which processes all accessible information on soils including biological analyses (vegetable and animal fragments, shells of soil microorganisms, pollen analyses, etc.) and anthropogenic relics (building materials, glass, slag, etc.) has been introduced. In future, most probably, genetic analysis will be used. But it is still necessary to carry out many studies both at the level of basic and applied research.

When pedological forensic traces are examined, usually two kinds of basic types of tasks requested. [1] Either it is a standard comparison. It means the questioned soil phases are compared with collected reference samples to confirm the crime scene location, a vehicle route, etc. Or it is requested to estimate an unknown location where the contamination with soil took place. In both cases it is a complex analysis. The analysis system allows carrying out a qualified examination of soil relics on clothing, shoes, human body, etc. and comparison of these traces with reference soil samples. The methods can be used also to detect presence of some important phases even in a heavily contaminated traces originating from different sources (e.g. composite sample obtained from soil-contaminated carpets of questioned vehicle, etc.). [2] Lately, automated mineralogical analysis has enlarged the basic analyses system. It is to get statistical data for subsequent comparison on one side and because it is a method less requiring an expert with a longterm practice. Systems of automated mineralogical analysis are based on adapted electron microscopes equipped with 2 or 4 EDS detectors. The systems are offered by at least two world SEM producers and are used especially in mining industry. The principle is rather simple. The sample in the form of a polished section of a rock, core sample or embedded grains is inserted into a special holder. The sample's surface (usually with diameter $25 \mathrm{~mm}$, but can be even larger) is divided into quadrants from which, point by point, both BSE signal and the EDS spectrum are scanned and evaluated. [3] Two or four EDS detectors are, within the standard procedure, applied especially to obtain a better signal. Automated classification of mineral phases is executed based on the obtained data and using either the producer's or user's databases.

The systems are designed to process polished planar samples. However, this method is not very suitable for forensic soils analysis. Firstly, the preparation of the polished sections takes some time and, secondly, it would require the sample to be divided into the individual grain size fractions. Afterwards, polished sections would be prepared from the fractions. That is why experiments were carried out in cooperation with the producer which were to find a direct way to analyze grains with topographic surfaces. The tests started with synthetic samples of known mineral phases which were placed in predefined positions. Then optimal analysis parameters were sought as well as the whole system setting geometry and software adoptions were made.

A new set was successfully developed. It significantly eliminated the influence of the topographic surface. Further, a whole range of tests on measurement reproducibility was performed. It means, an identical set of sampled grains was analyzed and then the same sample was scanned while being turned by 15 degrees. Then, repeatedly, the sampled sets of grains from an identical sampling location were scanned as well. These experiments were repeatedly performed on different samples. 
Repeatability and reproducibility of measurements proved to be very good. For example, for percent by weight of mineral phases representation, with 5-times-repeated measurement of the same set of untreated grains with a topographic surface, the standard deviation for the individual phases measurement was under 0.01 . When the samples were rotated by 15 degrees, with 7 repetitions, the deviation was maximally 0.28 . For repeated measurements, with samples collected at the same location, the deviation reached 0.45 maximally.

A few dozens of similar experiments were carried out. Of course, it would be possible to continue to the more detailed specification e.g. "compounds Fe, O, OH" or "K, Al, Si compound" (confirmation of, for example, probable sericite) but most of the soil analyses in the forensic fields concerns a relative mutual comparison of samples/traces while the experiments performed illustrate a very good reproducibility of results.

Other experiments with real samples of soil phases proved that the differences can be statistically evaluated even concerning the samples collected from locations that are close to each other. Depending on the location, exposition and vegetation at the location, provable and repeatedly measurable differences can be found even if the samples were taken at the locations as close as approximately $15 \mathrm{~m}$. It is because not only the bedrock content but also vegetation cover, ongoing weathering processes in soil and other factors also have crucial influence on confirmed mineralogical content.

Automated analysis does not seek to substitute the whole complex system of forensic soil analysis but it constitutes its suitable supplement. [4]

\section{References}

[1] Kotrlý M.: Analysis of pedological traces in forensic practice and new possibilities in this field. Microsc. Microanal. 22 (Suppl 3), 2016.

[2] Kotrlý M.: New Possibilities of Analysis and Comparison of Soil Phases in Forensic Practice. 8th European Academy of Forensic Science Conference. Lyon, 2018.

[3] Kotrly M.: Capability of Electron Microscopy in Forensic Science. Microsc. Microanal. 24 (Suppl 1), 2018.

[4] Microanalytical methods at ICP were supported by projects of Ministry of the Interior VI20152020035, VI20192022148 